\title{
Dissecting Halo Components in IFU Data
}

\author{
Michael Merrifield ${ }^{1, *}$, Evelyn Johnston ${ }^{2}$ and Alfonso Aragón-Salamanca ${ }^{1}$ \\ 1 School of Physics \& Astronomy, University of Nottingham, Nottingham NG7 2RD, UK; \\ alfonso.aragon@nottingham.ac.uk \\ 2 European Southern Observatory, Alonso de Córdova 3107 Vitacura, Casilla 19001, Santiago de Chile, Chile; \\ ejohnsto@eso.org \\ * Correspondence: michael.merrifield@nottingham.ac.uk; Tel.: +44-115-951-5186
}

Academic Editors: Duncan A. Forbes and Ericson D. Lopez

Received: 20 April 2017; Accepted: 23 May 2017; Published: 25 May 2017

\begin{abstract}
While most astronomers are now familiar with tools to decompose images into multiple components such as disks, bulges, and halos, the equivalent techniques for spectral data cubes are still in their infancy. This is unfortunate, as integral field unit (IFU) spectral surveys are now producing a mass of data in this format, which we are ill-prepared to analyze effectively. We have therefore been developing new tools to separate out components using this full spectral data. The results of such analyses will prove invaluable in determining not only whether such decompositions have an astrophysical significance, but, where they do, also in determining the relationship between the various elements of a galaxy. Application to a pilot study of IFU data from the cD galaxy NGC 3311 confirms that the technique can separate the stellar halo from the underlying galaxy in such systems, and indicates that, in this case, the halo is older and more metal poor than the galaxy, consistent with it forming from the cannibalism of smaller satellite galaxies. The success of the method bodes well for its application to studying the larger samples of cD galaxies that IFU surveys are currently producing.
\end{abstract}

Keywords: galaxy formation and evolution; integral field unit spectroscopy; cD galaxies

\section{Introduction}

Our understanding of galactic structure and its evolution has been driven forward on a tidal wave of data, which has allowed us to systematically quantify the properties of these beautiful systems on the basis of very large surveys. The Sloan Digital Sky Survey (SDSS) produced imaging data on millions of galaxies, which, though an amazing resource, presented the additional challenge of how to quantify so much information in a meaningful form. Fortunately, tools such as GALFIT had been developed that allow the properties of the components that make up galaxies to be objectively quantified by simultaneously fitting a number of simple functions such as two-dimensional Sersic profiles to their images to quantify each component [1]. Such automated techniques can readily be applied even to surveys on the scale of SDSS, producing a mass of summarizing data on the disks, bulges, and other components that make up galaxies in the nearby Universe [2].

However, such imaging data has its limitations, as there are degeneracies and ambiguities when seeking to understand the stellar populations that make up each component if only broad-band colours are available. To address this problem, several large spectroscopic galaxy surveys such as SAMI [3] and MaNGA [4] are currently under way, using integral field units (IFUs) that produce a spectrum at each point on the sky across the face of each galaxy, generating a cube of data with a spectrum in the $z$-direction for every position $\{x, y\}$. From such spectral data, one can derive properties such as age and metallicity, and how they vary with position in each galaxy. Unfortunately, tools similar to those in common usage for imaging data do not exist for such data cubes, so we have been developing new ones specifically to decompose spectral data cubes of galaxies into their constituent components. Here, 
we describe the first application of such a technique to a $\mathrm{cD}$ galaxy in order to separate the spectral properties of its halo from the main elliptical component.

\section{Materials and Methods}

The essence of the new method we have developed is very simple. At every wavelength, an IFU data cube contains a narrow-band image of the galaxy at that wavelength, so the conventional machinery of image fitting and decomposition can be applied, yielding the amount of light in each component at that wavelength. Repeating the analysis for each such wavelength slice then gives the amount of light in each component as a function of wavelength, more conventionally described as the spectrum of each component. Since a complete data cube has been reduced to a few one-dimensional spectra, the resulting signal-to-noise ratios of the derived component spectra are very high, allowing them to be readily analyzed in terms of their stellar populations.

Of course, in practice things are more complicated. For one thing, each image slice must correspond to the same rest wavelength across the whole galaxy, so the shifts and broadening due to the galaxy's kinematics have to be allowed for. In addition, the fitting process occasionally produces unphysical results, particularly on the relatively low signal-to-noise ratio 'images' of single wavelength slices, so the fitting process can be quite unstable. Fortunately, there is the additional physical constraint that the structural parameters of a galaxy's components will not vary dramatically between adjacent wavelengths, so the fitting process can be regularized by imposing this additional constraint.

We have recently successfully implemented this approach in analyzing IFU data, and applied it to a study of S0 galaxies, deriving spectra for their bulge and disk components [5]. The results showed that the bulges were systematically younger than the disks, implying that the 'last gasp' of star formation occurred when gas was channeled into the centres of the systems as they were being stripped, meaning that, at least in a luminosity-weighted sense, the starlight of the bulges is younger than that of the surrounding disk [5]. Although initially applied to separating bulges and disks, the technique is completely general, and can be used to derive spectra for any photometrically-distinct components in a galaxy, so here we turn to separating elliptical cores from their surrounding stellar halos in $\mathrm{cD}$ systems, to explore their interrelationship.

An excellent target for this pilot study is offered by NGC 3311, the classic cD galaxy at the centre of the Hydra I Cluster. The centre of this cluster was the target for a set of four pointings with the large-area Multi-Unit Spectroscopic Explorer (MUSE) [6] at the Very Large Telescope (VLT) obtained under ESO Program 094.B-0711(A) (PI Arnaboldi); this archival dataset covered a significant fraction of the light from NGC 3311. Such an extensive observation of a nearby $\mathrm{cD}$ galaxy is complete overkill for the analysis that we wish to undertake here, but it provides the ideal test case as the high-quality data means that the results of our simple summarizing analysis can be compared with a more sophisticated study of the detailed data, to provide some confidence in the method when such unusually high-quality observations are not available.

\section{Results}

A sample image slice of the data set is presented in Figure 1, which shows the footprint of the four MUSE pointings, the image data in this single wavelength slice, the elliptical and halo components fitted as a de Vaucouleurs law and an exponential respectively, and the residuals once they are subtracted. There are significant negative residuals at small radii, associated with the documented unusually low surface brightness of the centre of this galaxy [7], but tests showed that the results were robust whether or not this region was included in the fit. There are also still minor artifacts in the data associated with the flatfielding of individual pointings, but again not at a level that should compromise the results unduly. 

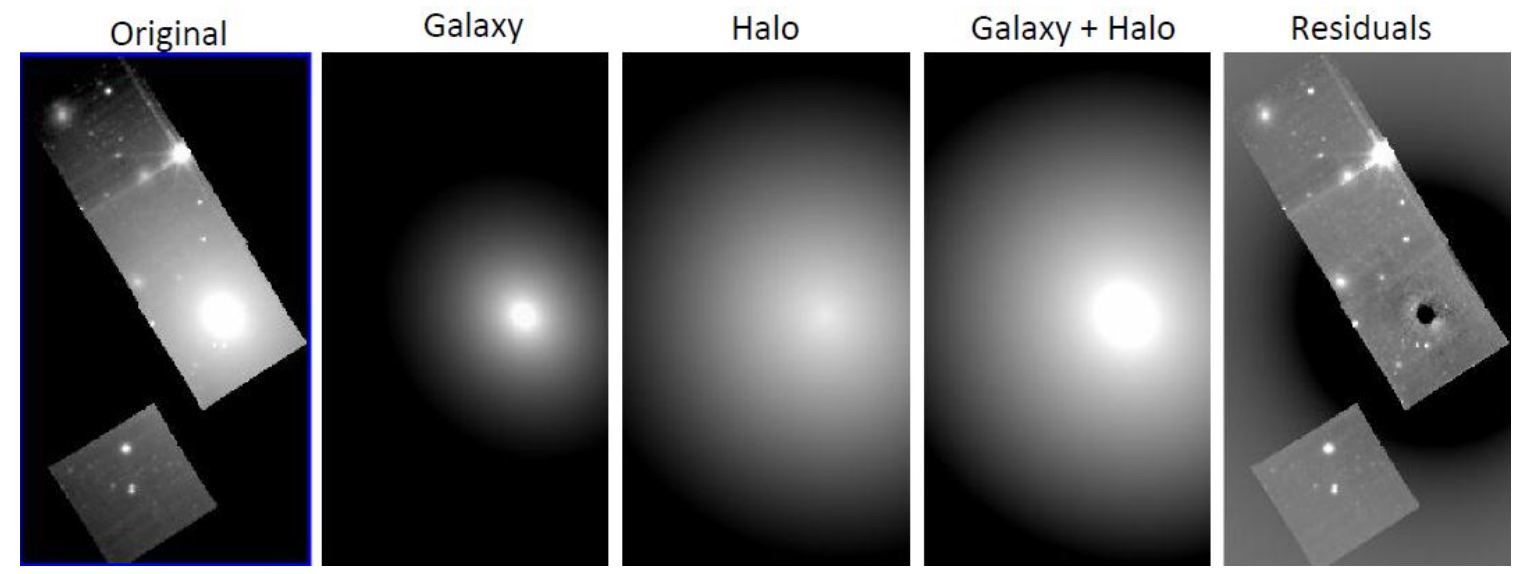

Figure 1. A sample image slice through the MUSE observations of NGC 3311, showing the footprint of the observations and image at a single wavelength, the model fit to these data, and the residuals left after the fitted model has been subtracted.

Having repeated this fitting process for all wavelength slices, as set out in Section 2, we analyzed the resulting preliminary spectra of the disentangled galaxy and halo components by determining various Lick indices from them. The results, together with an indicative grid of values characteristic of various single stellar population models, is presented in Figure 2. As is clear from this figure, both components are very old, with the halo apparently containing slightly older stars than the main body of the galaxy. More clear-cut, the halo is of significantly lower metallicity with $[\mathrm{Fe} / \mathrm{H}] \sim-0.4$, as compared to the galaxy's value of $\sim+0.1$. The latter value is characteristic of a massive elliptical galaxy, but the halo value is what one would expect of a galaxy that is a factor of $\sim 10$ less luminous [8] (Figure 8). This fits well with a scenario in which all the stars that make up this galaxy formed early, but the halo component was originally in smaller satellite galaxies that formed their stars slightly earlier than the central system, which were subsequently accreted by the central galaxy in a series of minor mergers.

Further evidence for the early formation of all the stars that ultimately make up NGC 3311 comes from the alpha enhancement measured in both components (see Figure 2b). Such enhancement occurs when star formation is complete on a timescale shorter than the point at which Type 1a supernovae start to occur, so the only heavy nuclei that have been recycled into stars are from the Type 2 supernovae that produce alpha elements in abundance. Thus, we know that the stars making up both galaxy and halo must have been formed in a single short burst rather than being spread over the lifetime of the system.

In this test case, the data are of high enough quality that one can see crudely how these indices vary with projected radius in the raw spectra. The results are shown as the small points in Figure 2; reassuringly, these results are consistent with previous study of the variation in the projected stellar population with radius [9]. As can be seen, the Lick indices generally show the kind of gradient with a radius that one would expect if the observed light were made up of the derived galaxy and halo components, whose relative weight changes with the radius such that the total spectrum transitions smoothly from galaxy-dominated at small radii to halo-dominated at large radii. The only clear exception to this consistent picture is at very small radii where the $\mathrm{H} \beta$ absorption-line index is consistently low, reflecting the presence of gas at the centre of this system, whose emission lines partially fill in the $\mathrm{H} \beta$ absorption line. Note, however, that the fitting process effectively rejects this discrepant data, making the analysis presented here robust against such contaminants, and would do so even in cases where we did not have data of the quality required to identify them explicitly, as we benefit from here. 


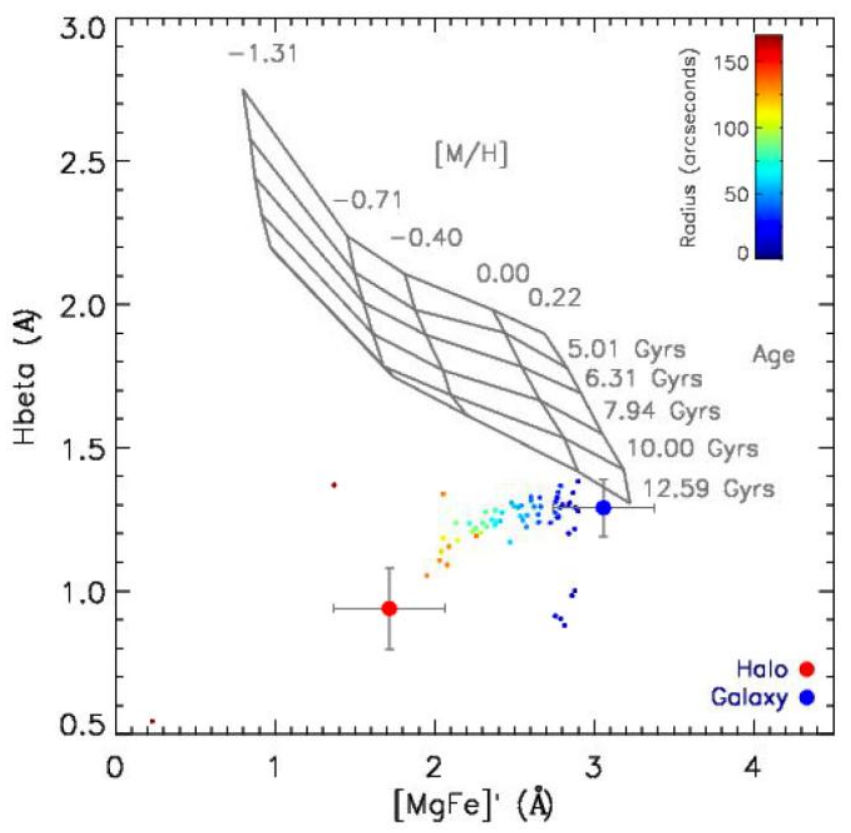

(a)

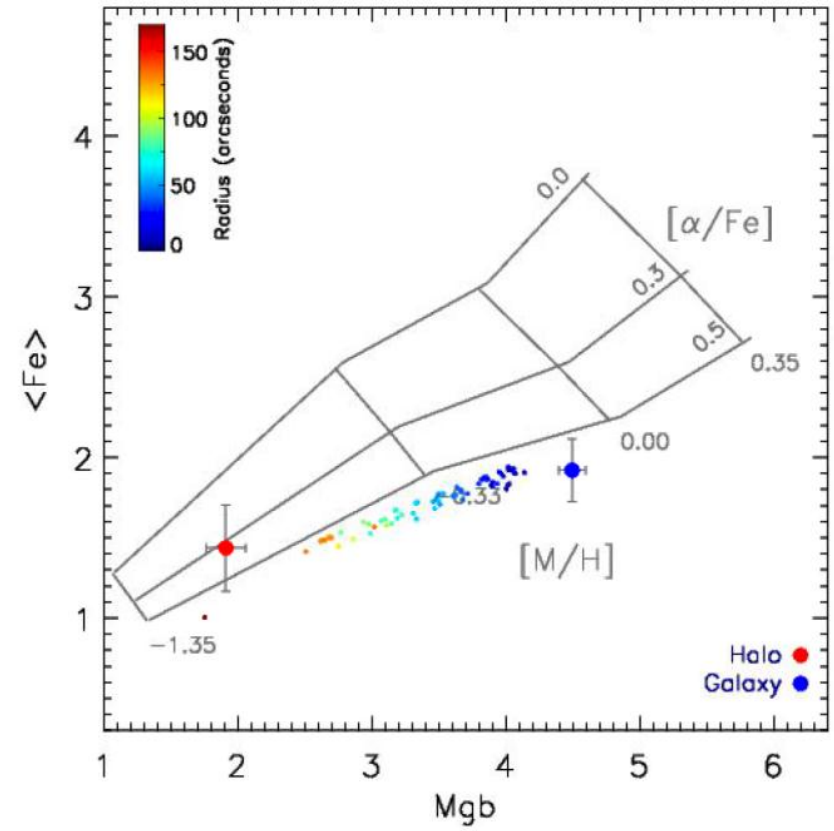

(b)

Figure 2. Stellar population models superimposed on Lick indices measured from decomposed galaxy and halo: (a) age and metallicity; (b) metallicity and alpha-element enhancement. The large points show the values from decomposed components; the small points show values derived as a function of projected radius directly from the data.

\section{Discussion}

In this paper, we have shown how spectral data cubes can be analyzed in a manner analogous to image data, decomposing a galaxy into separate spectra of its photometric components. A preliminary pilot study of IFU spectral observations of the cD galaxy NGC 3311 indicates how this system can be decomposed into a main galaxy component and a surrounding stellar halo; both components are made up of old rapidly-formed stellar populations, whose properties are consistent with the main component forming in situ, while the halo was acquired from mergers with multiple smaller satellite systems. 
More generally, the success of this analysis in reproducing the results of more detailed analysis of high-quality data bodes well for its application to smaller, lower-quality data cubes. In addition, the automated robust nature of the fitting process means that it is well suited to an objective analysis of larger samples of $\mathrm{cD}$ galaxies. We therefore look forward to its application to more extensive survey data in the coming years, to see how the formation histories of the extended stellar halos around $\mathrm{cD}$ galaxies might depend on other properties of these systems.

Acknowledgments: The authors wish to thank the organizers of the excellent conference, "On the Origin (and Evolution) of Baryonic Halos", which motivated this work, and at which it was presented. The results presented here are based on observations collected at the European Organisation for Astronomical Research in the Southern Hemisphere under ESO programme 094.B-0711(A).

Author Contributions: E.J. carried out the analysis presented here; A.A.-S. contributed his expertise on the interpretation of line indices; M.M. suggested the project and wrote the paper.

Conflicts of Interest: The authors declare no conflict of interest.

\section{References}

1. Peng, C.Y.; Ho, L.C.; Impey, C.D.; Rix, H.-W. Detailed structural decomposition of galaxy images. Astron. J. 2002, 124, 266-293. [CrossRef]

2. Meert, A.; Vikram, V.; Bernadi, M. A catalogue of 2D photometric decompositions in the SDSS-DR7 spectroscopic main galaxy sample: Preferred models and systematics. Mon. Not. R. Astron. Soc. 2015, 446, 3943-3974. [CrossRef]

3. Bryant, J.J.; Owers, M.S.; Robotham, A.S.G.; Croom, S.M.; Driver, S.P.; Drinkwater, M.J.; Lorente, N.P.F.; Cortese, L.; Scott, N.; Colless, M.; et al. The SAMI Galaxy Survey: Instrument specification and target selection. Mon. Not. R. Astron. Soc. 2015, 447, 2857-2879. [CrossRef]

4. Yan, R.; Bundy, K.; Law, D.R.; Bershady, M.A.; Andrews, B.; Cherinka, B.; Diamond-Stanic, A.M.; Drory, N.; MacDonald, N.; Sánchez-Gallego, J.R.; et al. SDSS-IV MaNGA IFS Galaxy Survey-Survey Design, Execution, and Initial Data Quality. Astron. J. 2016, 152, 197. [CrossRef]

5. Johnston, E.J.; Häußler, B.; Aragón-Salamanca, A.; Merrifield, M.R.; Bamford, S.; Bershady, M.A.; Bundy, K.; Drory, N.; Fu, H.; Law, D.; et al. SDSS-IV MaNGA: Bulge-disc decomposition of IFU data cubes (BUDDI). Mon. Not. R. Astron. Soc. 2017, 465, 2317-2341. [CrossRef]

6. Bacon, R.; Accardo, M.; Adjali, L.; Anwand, H.; Bauer, S.; Biswas, I.; Blaizot, J.; Boudon, D.; Brau-Nogue, S.; Brinchmann, J.; et al. The MUSE second-generation VLT instrument. Proc. SPIE 2010, 7735. [CrossRef]

7. Vasterberg, A.R.; Lindblad, P.O.; Jorsater, S. An optical study of the cD galaxy NGC3311 and the giant elliptical galaxy NGC3309 in the cluster Hydra I. Astron. Astrophys. 1991, 247, 335-347.

8. Chilingarian, I.V.; Mieske, S.; Hilker, M. Dynamical versus stellar masses of ultracompact dwarf galaxies in the Fornax cluster. Mon. Not. R. Astron. Soc. 2011, 412, 1627-1638. [CrossRef]

9. Barbosa, C.E.; Arnaboldi, M.; Coccato, L.; Hilker, M.; Mendes de Oliveira, C.; Richtler, T. The Hydra I cluster core. I. Stellar populations in the cD galaxy NGC 3311. Astron. Astrophys. 2016, 589, A139. [CrossRef] 